\title{
De tradiciones y métodos: investigaciones pictográficas
}

\author{
Michel R. Oudijk
}

La investigación de los documentos pictográficos se ha desarrollado mucho durante las últimas décadas. Aunque recientemente las diferencias son cada vez menos claras, en este ámbito de estudio se pueden reconocer cinco escuelas y tradiciones de investigación, cada una con su propio objetivo y metodología. En el siguiente texto se presenta una descripción general de las escuelas y tradiciones con unas reflexiones sobre sus métodos. Sin pretender ser exhaustivo por el limitado espacio, el objetivo es dar una idea sobre el desarrollo de la investigación e indicar algunos problemas y directrices para futuros estudios.

PALABRAS CLAVE: pictografía mesoamericana, método de investigación

\begin{abstract}
About Traditions and Methods: Pictographic Research
Research on pictographic documents has strongly evolved during the last few decades. Even though their differences have become less clear, five schools of thought can be distinguished, each one with its own objectives and methodologies. This paper makes a general description of the schools and traditions on this field, and reflects on their methods. Without pretending to be an exhaustive analysis, due to space constraints, the author's goal is to shed some light on the evolution of research and to point out some problems and a few guidelines for future studies.
\end{abstract}

KEY WORDS: Mesoamerican Pictography, Research Method

MICHEL R. OUDIJK: Instituto de Investigaciones Filológicas, Universidad Nacional Autónoma de México, México

Desacatos, núm. 27, mayo-agosto 2008, pp. 123-138.

Recepción: 20 de noviembre de 2007 / Aceptación: 10 de enero de 2008 
$\mathrm{D}$ urante las últimas tres décadas hemos visto un desarrollo abrumador en los estudios de los documentos pictográficos mesoamericanos. Si tomamos en cuenta solamente la productividad, vemos que desde 1975 se ha escrito una enorme cantidad de trabajos que superan lo realizado durante los cien años anteriores. Los investigadores tienen ahora acceso a más reproducciones de documentos, lo que facilita enormemente la investigación, siendo Internet un medio y una fuente que todavía no se ha aprovechado en su máximo potencial. Aunque aún falta muchísimo por hacer, no hay duda de que los avances nos han acercado a un mejor entendimiento de los mensajes plasmados por los tlacuilos o pintores de los libros indígenas.

Como en todos los demás estudios mesoamericanos, el análisis de los documentos pictográficos es hoy día una disciplina que sigue ciertas reglas metodológicas, con el objetivo de evitar resultados disparatados como los de Agustus Le Plongeon o las conclusiones astralistas de Eduard Seler.

Para realizar el análisis de los documentos pictográficos, al igual que cualquier otro documento, es necesario aplicar un método estricto. El método nos indica qué pasos analíticos tiene que seguir un investigador para llegar a una interpretación y conclusión sobre el texto pictográfico. Así, el método es nuestra guía y señala al investigador y al lector qué interpretación es aceptable y por qué, cuál asociación es legítima y por qué, etc. También nos indica cuáles son nuestros límites de análisis. Sin un método sólido, cualquier análisis es legítimo y, por tanto, cualquier resultado es aceptable, lo cual, obviamente, no es el caso. Aunque los elementos pictográficos pueden ser polivalentes y la lectura de un texto pictográfico puede variar en ciertos aspectos, el mensaje de un documento siempre es uno. Esto quiere decir que no importa el método que uno aplique para analizar un documento; al final, la identificación del mensaje debe ser la misma, aunque la lectura de las distintas escenas pueda variar en su riqueza.

Un grave problema en los estudios de los documentos pictográficos mesoamericanos ha sido que muy pocos investigadores han indicado el método con que analizan el documento o el grupo de documentos. En algunos casos podemos inferir el método de análisis por medio de una lectura precisa del texto. Sin embargo, si el método no es explícito, es muy difícil determinar cuáles son los límites analíticos dentro del estudio que estamos leyendo, o si el autor es consciente de esos límites. El problema está, entonces, en no poder determinar en qué se basa una interpretación y, consecuentemente, en ser incapaz de determinar si dicha interpretación es válida o no. Como una de las bases de los estudios académicos es la posibilidad de verificación de los resultados, es necesario indicar y precisar los pasos metodológicos.

Actualmente existen varias 'escuelas' y 'tradiciones' que aplican diferentes métodos de investigación. El término 'tradición' no necesariamente se refiere a un grupo de investigadores que trabajan o han trabajado juntos, o que se han puesto de acuerdo sobre cómo trabajar con los documentos pictográficos. Más bien, se trata de un grupo que trabaja más o menos de la misma manera, sea por inspiración intelectual de algún investigador, sea por contacto directo. Con 'escuela' me refiero a un grupo de investigadores que han trabajado juntos según líneas teóricas y metodológicas explícitas. Actualmente, y de manera general, podemos identificar cinco tradiciones y escuelas diferentes: la escuela holandesa, la tradición mexicana, la tradición americana, la escuela española y la escuela galarcista. Primeramente haré un recorrido histórico de estos grupos, seguido por un análisis de sus métodos. Por razones de espacio trataré exclusivamente de investigadores todavía activos. Tampoco puedo tratar a todos los investigadores que han escrito algo sobre los documentos pictográficos, porque eso requeriría un trabajo mucho más extenso que el presente. Sólo intentaré identificar algunas tendencias que se pueden reconocer dentro del campo de los estudios pictográficos durante la segunda mitad del siglo XX.

\section{LA ESCUELA HOLANDESA ${ }^{1}$}

En varias de sus publicaciones, la escuela holandesa ha hecho explícito su método de trabajo (Jansen, 1982, 1988;

\footnotetext{
${ }^{1}$ Kevin Terraciano ha denominado este enfoque como la 'escuela holan-
} 
Loo, 1982, 1987; Doesburg, 2001; Roskamp, 1998; Oudijk, 2000; Asselbergs, 2004). El método, llamado etno-iconología, tiene su origen en el esquema metodológico de Erwin Panofsky, pero con ciertas adaptaciones para el análisis de los documentos pictográficos mesoamericanos.

El primer nivel de la etno-iconología es aquel en el que se identifican los elementos pictográficos. Dentro de la tradición pictórica mesoamericana nunca se representaba un elemento aislado, sino siempre en un conjunto con relaciones significantes, por lo que, consecuentemente, tenemos que identificarlos de esa misma manera. Un ejemplo del Códice Selden (pp. 5-I) aclara el procedimiento:

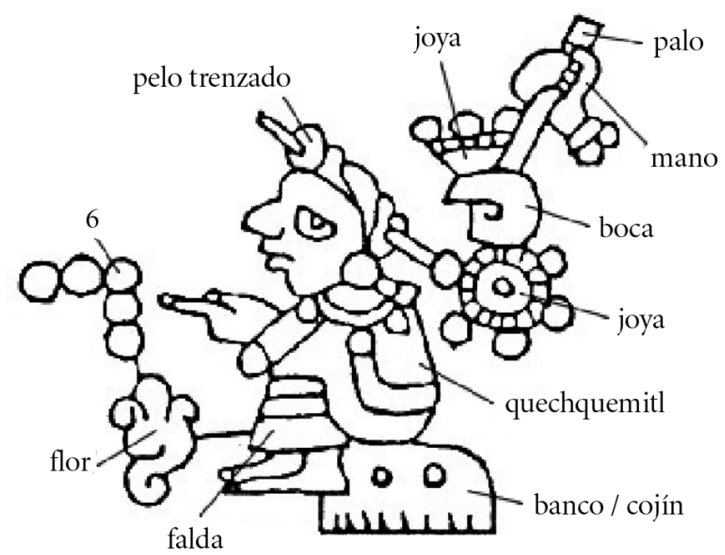

La identificación de los elementos mínimos se puede hacer con base en la comparación con otros documentos pictográficos, documentos alfabéticos coloniales y tradiciones indígenas actuales. El hecho de que este personaje lleve la combinación del pelo trenzado, un quechquemitl y una falda, le identifica como una mujer. Normalmente, en los códices históricos los personajes van acompañados por su nombre calendárico, que aquí po-

desa' por la participación de muchos investigadores de la Universidad de Leiden (Maarten Jansen, Aurora Pérez Jiménez, Peter van der Loo, Roswitha Manning, Sebastián van Doesburg, Hans Roskamp, Florine Asselbergs y Michel R. Oudijk) en el desarrollo y diseño del método. Sin embargo, ha sido un trabajo en conjunto que también comprendió a investigadores de otras instituciones como Luis Reyes García, Ferdinand Anders, Mary Elizabeth Smith, Nancy Troike, María Castañeda de la Paz, Manuel Hermann Lejarazu y Ubaldo López García. demos identificar como 6-Flor. Su nombre personal está representado por el complejo conjunto de glifos detrás de la señora y que contiene un elemento que representa una joya, un palo, una mano y una boca. Las joyas son muy comunes en los nombres femeninos de los documentos mixtecos y expresan algo 'precioso'. Finalmente, está sentada sobre un banco o cojín con piel de jaguar, lo que la identifica como gobernante de algún lugar.

El primer nivel interpretativo está ligado al segundo por medio de un subnivel que consiste en la determinación del género del manuscrito, con el fin de interpretar los diferentes elementos que componen una escena pictográfica en su propio contexto. Es decir, que un templo en un manuscrito religioso puede tener un significado diferente al que tiene en un texto histórico. Muchos de los documentos pictográficos mesoamericanos son del género histórico-geográfico. Esto no quiere decir que los documentos mismos nunca hayan sido objeto de veneración como es, por ejemplo, el caso del Lienzo de Petlacala de Guerrero, el cual es ritualmente apreciado (Oettinger y Horcasitas, 1982). Tampoco significa que los elementos representados o sus escenas no pudiesen tener un significado religioso. Obviamente, si la fundación de un pueblo o su linaje está representado en él, esto tenía y tiene todavía una fuerte connotación religiosa para el grupo en cuestión. De hecho, los gobernantes y sus ancestros eran realmente personas divinizadas ya que descendían de un fundador épico nacido de un árbol, una roca o un río. Debemos entonces considerar a las genealogías que queremos leer como una historia indígena de carácter divino.

El segundo nivel interpretativo consiste en un enfoque temático, en el que los conjuntos de elementos son interpretados mediante asociaciones significativas dentro de su género. Uno de los componentes principales de la etno-iconología es el uso del enfoque histórico directo, basado en la continuidad cultural de sociedades indígenas en Mesoamérica, pero con particular atención en el fenómeno de la disyunción. Es decir, la identificación de la relación entre el significado y el significante. Según Panofsky, el uso de cierto símbolo - o en este caso, cierto elemento pictográfico- puede perdurar a través del tiempo, pero no siempre con el mismo sentido. Quiere 
decir que la relación entre el significado y el significante puede cambiar dentro del proceso que llamamos disyunción. Fue George Kubler $(1961,1981)$ quien argumentó que precisamente la disyunción hace imposible el uso de la analogía como instrumento para la interpretación del pasado mesoamericano. Sin embargo, Henry B. Nicholson (1976) y Peter van der Loo (1987) han demostrado que sí se puede aplicar la analogía, pero solamente dentro un análisis cuidadoso y riguroso por medio de las unidades temáticas. por ejemplo, una de un contexto religioso huichol del siglo XVI. Por otro lado, una analogía será más fuerte si se comparan dos unidades temáticas con muchos elementos. Una analogía entre un solo elemento nunca es válida porque no hay contexto ni relación significativa. Una analogía funciona cuando se pueden mostrar las mismas relaciones significativas entre varios elementos de dos unidades temáticas. Un ejemplo del Códice Bodley puede aclarar el asunto:

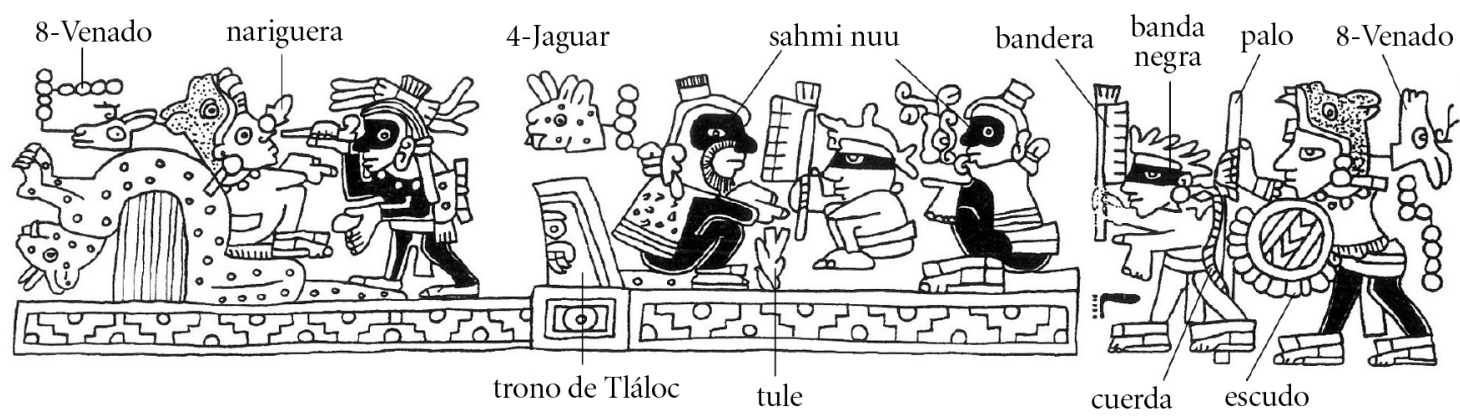

Una unidad temática es un conjunto de elementos con ciertas relaciones significativas. La analogía se aplica usando la unidad temática de un contexto conocido para entender otra unidad temática similar en un mismo contexto menos conocido. Normalmente, en el enfoque histórico directo el contexto conocido es el que existe en los pueblos indígenas actuales, mientras que el contexto menos conocido es el que existió en el pasado. Sin embargo, también podemos imaginar una analogía entre un contexto histórico reciente en relación con un contexto histórico más lejano. Es fundamental enfatizar la importancia de la similitud de los contextos comparados. En general podemos considerar que, mientras más alejados en el tiempo o en el espacio estén los dos contextos, menos convincente será la analogía. Es decir, si comparamos una unidad temática de un contexto religioso mixteco actual con una del inicio de siglo XX del mismo contexto, es más probable que ésta sea más convincente que una analogía entre esa primera unidad temática y,
La lectura es de la derecha hacia la izquierda. Vemos a un señor llamado 8-Venado que tiene en su mano un palo que indica que viene de un largo camino. En la otra mano lleva un escudo con chevrones, lo que indica que es un guerrero, y una cuerda con la que está atado un señor casi desnudo con una banda negra en los ojos y una bandera en su mano. Todos estos elementos indican que éste es un cautivo a quien van a sacrificar. Se dirigen hacia un lugar donde un señor barbado llamado 4-Jaguar, que está sentado en un trono con la máscara de Tláloc, recibe a otro señor sentado, quien le presenta otro cautivo. Ambos señores tienen los ojos negros. Estamos ante un juego de palabras mixteco que se refiere a los sahmi nuи o 'náhuatl hablantes', y que se representa pictográficamente mediante 'ojos quemados' o sami nuu. La escena quiere decir que el señor 4-Jaguar era gobernante de un señorío nahua que está identificado por un tule y que se lee como Tollan. Después de la entrega de los cautivos, el señor 8-Venado se echó hacia atrás sobre una piedra 
cubierta con una piel de jaguar donde recibió una nariguera de un señor pintado de negro, color para indicar que era un sacerdote, y que acaba de perforarle la nariz.

Esta gran escena que podemos identificar como una unidad temática se puede entender por medio de una analogía con otra unidad temática colonial incluida en la Relación Geográfica de Cholula:

[...] los tales reyes y caciques, en heredando el reino o senoorío, venían a esta ciudad a reconocer obediencia al ídolo de ella, Quetzalcóatl, al cual ofrecían plumas ricas, mantas, oro y piedras preciosas, y otras cosas de valor. Y, habiendo ofrecido, los metían en una capilla que para este efecto estaba dedicada, en la cual los dos sumos sacerdotes los señalaban horadándoles las orejas, o las narices o el labio inferior, según el señorío que tenían. Con lo cual quedaban confirmados en sus señoríos, y se volvían a sus tierras [...] (Acuña, 1984-1985, II: 130-131).

La misma relación de Cholula explica que la ciudad también se llamaba Tollan Cholollan (ibid.: 128) y, obviamente, era un señorío nahua. El señor 8-Venado era gobernante de Tilantongo en la Mixteca Alta, de donde vino andando hasta llegar a Cholula. Aunque el texto de la relación refiere a ciertos productos que fueron presentados delante del bulto del dios Quetzalcóatl, en el Códice Bodley vemos la presentación de cautivos delante del señor-sacerdote 4-Jaguar. Es interesante mencionar que, en el Códice Nuttall, este mismo 4-Jaguar está representado con una barba, una buba en la nariz y la pintura facial negra de líneas semicirculares, todos elementos asociados con Quetzalcóatl (Jansen, 1996). Parece entonces que 4-Jaguar era un sacerdote de este mismo dios y, como tal, una personificación de Quetzalcóatl mismo. Después de la entrega de los cautivos por 8-Venado, unos sacerdotes le perforaron la nariz y le pusieron una nariguera como símbolo de gobernante tolteca.

Las dos unidades temáticas son mutuamente explicativas, ya que algunos elementos del ritual de la nariguera no se mencionan en la Relación Geográfica de Cholula, pero los vemos representados en el Códice Bodley, y viceversa. Hay que mencionar que la identificación de este Tollan como Cholula, así como otros elementos de la unidad temática pictográfica, es posible gracias a un análi- sis de otros documentos pictográficos como, por ejemplo, el Códice Nuttall, el Códice Colombino y la Historia Tolteca-Chichimeca. Allí vemos que las relaciones significativas entre los diferentes elementos de las unidades temáticas son las mismas: la presentación de productos ante el dios Quetzalcóatl o su sacerdote en Tollan Cholula, para después perforar la nariz (para poner una nariguera), lo cual confirma el estatus del gobernante. La analogía entre las dos unidades es muy fuerte y por esa razón podemos suponer y trasponer ciertos elementos de una unidad a otra. Por ejemplo, sabemos que la piedra cubierta con la piel de jaguar sobre la cual está echado 8-Venado está situada en un templo, como se puede leer en la Relación Geográfica, aunque el templo no esté representado en el Códice Bodley². Asimismo, sabemos que la horadación de la nariz sirve para meter una nariguera (y la del labio para meter un bezote); si bien la nariguera no fue mencionada en la Relación Geográfica, sí está representada en el Códice Bodley.

De manera similar se trabaja con los múltiples documentos pictográficos histórico-geográficos en los cuales se registran los linderos que definen el territorio de una comunidad. En las pictografías, estos lugares pueden ser identificados únicamente como mojoneras si aparecen en el mismo orden que en su realidad geográfica y cuando existe la misma secuencia de más de dos mojoneras en las dos unidades temáticas. Así, en el Lienzo de Guevea es posible identificar Isoguatenco, Ticuatepequeg y Cosmaltepequez como tres mojoneras de Guevea porque exactamente en ese mismo orden aparecen en un mapa del siglo XIX y en el paisaje actual (fig. 1, p. 128). Por esta misma razón no es posible identificar Ticuatepequeg como el pueblo de Tehuantepec, porque los pueblos de Isoguatenco y Cosmaltepequez simplemente no existen y nunca han existido en el Istmo.

\footnotetext{
2 El Códice Colombino (pp. XIII) muestra una impresionante escena paralela de la perforación de la nariz de 8-Venado en la que el ritual tiene lugar en un templo de perlas en Tollan (Cholula). Este mismo templo de Cholula está representado en el Códice Bodley (folio 12-I) y probablemente es el mismo que está dibujado en la Historia ToltecaChichimeca (ff. 26v-27r). Véase también la perforación de la nariz del sucesor de 8-Venado, el señor 4-Viento, en Tollan Cholula (Códice Bodley, 34 IV-V).
} 


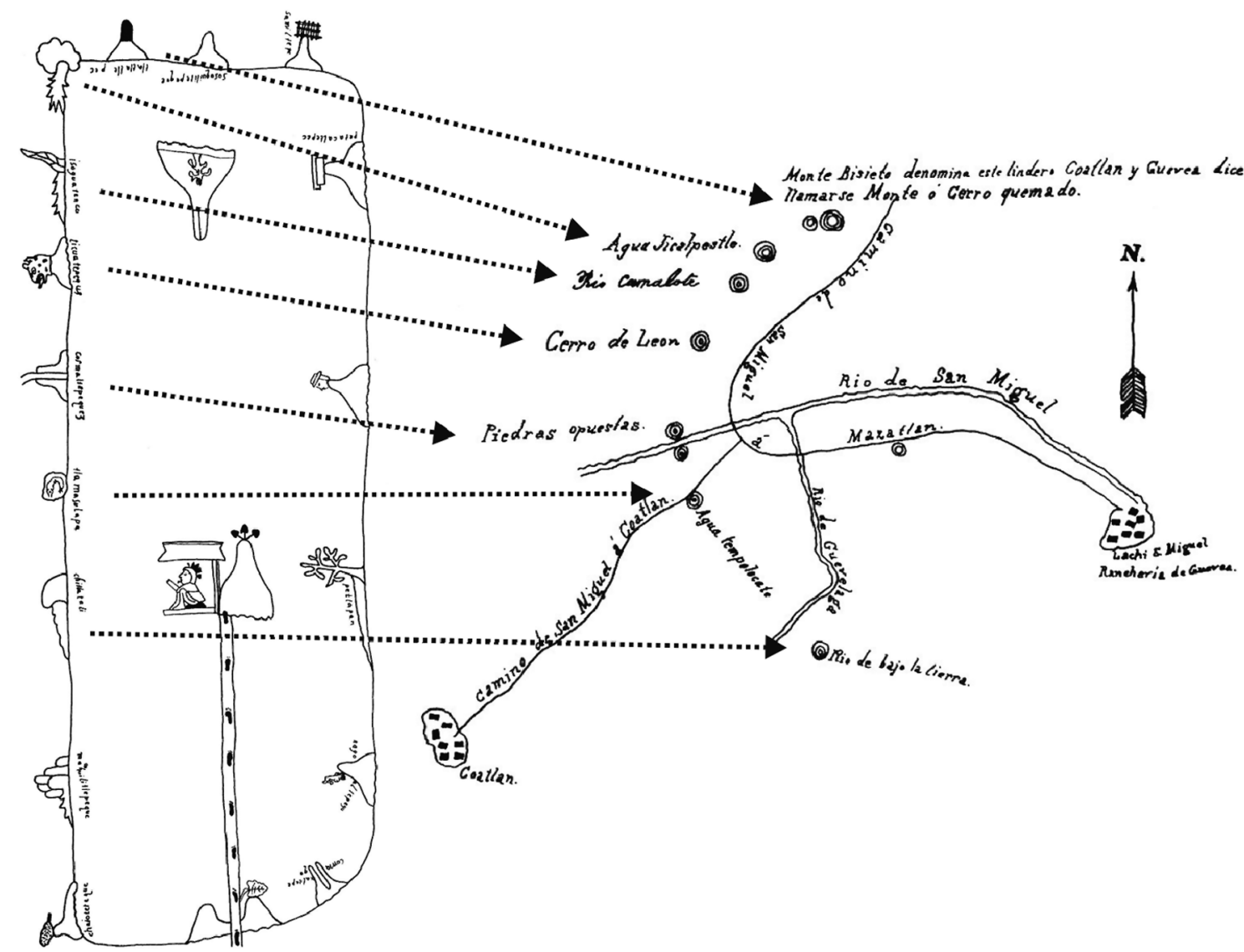

Figura 1. Mapa del lindero entre Guevea de Humboldt y Coatlán y Mazatlán (AGEO/CLT, leg. 76, exp. 38, ffs. 24-26, 1889). Comparación de las mojoneras en la parte superior del Lienzo de Guevea con las de un mapa de Guevea de Humboldt de 1889.

Queda claro que para aplicar el enfoque histórico directo y llegar a conclusiones sobre los documentos prehispánicos y coloniales es necesario un conocimiento profundo de los pueblos indígenas actuales. Este enfoque se basa en la continuidad cultural de las sociedades indígenas en Mesoamérica. Obviamente, dicha continuidad cultural no quiere decir que éstas son o fueron estáticas a través del tiempo. Al contrario, por definición una cultura viva nunca es estática, sino que siempre se encuentra en un desarrollo basado en sus raíces históricas. Si en nuestras investigaciones tomamos en cuenta y analizamos el proceso de cambio que sufren y han sufrido las sociedades indígenas durante su historia, es posible llegar a conclusiones con base en los pueblos actuales (aplicando las unidades temáticas, claro).
En este sentido es importante dar los ejemplos de Karl Anton Nowotny (1961) y Peter van der Loo (1987). En una publicación extraordinaria, Nowotny argumenta que los códices 'calendáricos' o 'religiosos' que forman el llamado Grupo Borgia son en verdad documentos mánticos que tienen una función de pronóstico y adivinación. Así, con base en la obra del etnólogo Leonhard Schultze-Jena (1938) sobre los manojos contados entre los tlapanecos, Nowotny (1961: 272-275) demuestra que los folios de los códices mánticos prehispánicos que contienen barras y puntos tratan de ofrendas contadas en peticiones rituales (fig. 2). En 1987, Van der Loo, con base en trabajo de campo en comunidades tlapanecas, mixes y nahuas, continuó el trabajo de Nowotny al demostrar la fuerte relación que, a lo largo del tiempo, existe entre los códices mánticos y 

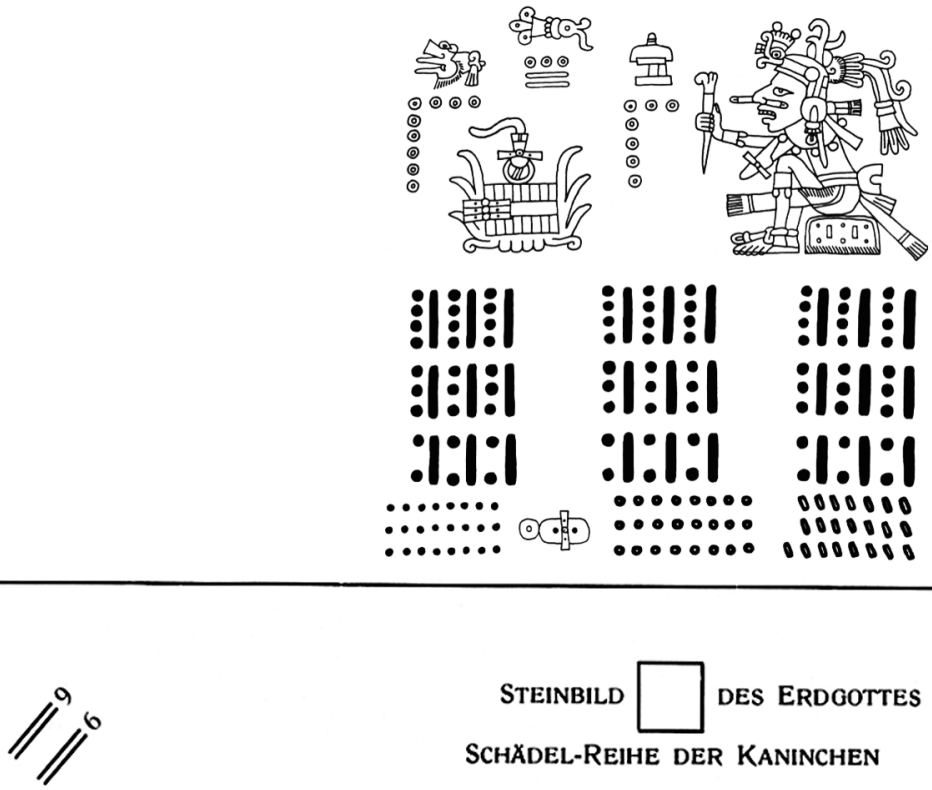

STEINBILD

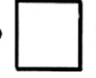

DES ERDGOTTES

SCHADEL-REIHE DER KANINCHEN

SCHADEL-REIHE DER HIRSCHKUHE

SCHĀDEL-REIHE DER MANNLICHEN HIRSCHE
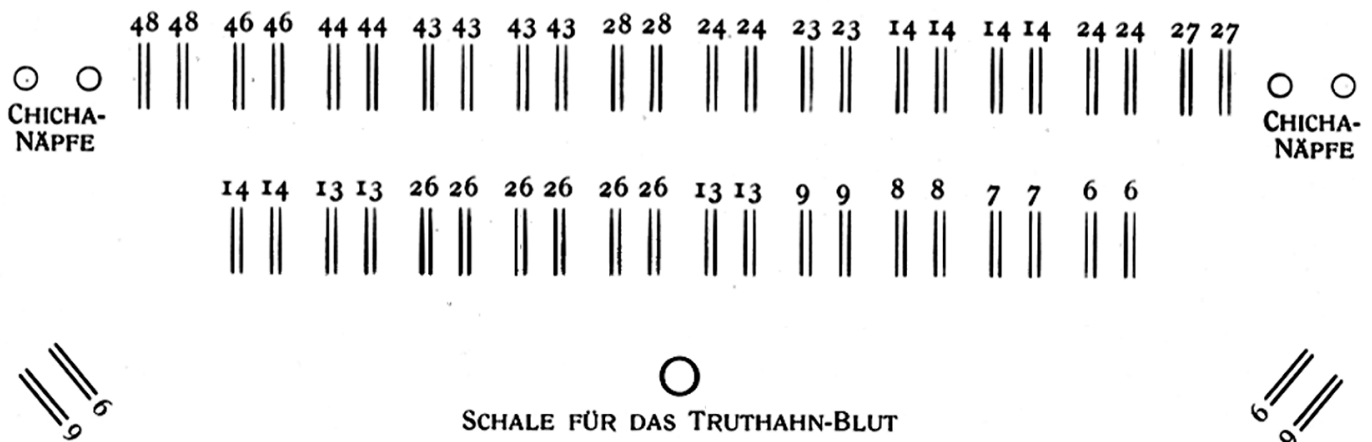

SCHALE FUR DAS TRUTHAHN-BLUT

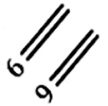

Figura 2. Peticiones rituales con ofrendas contadas en el Códice Fejervary-Mayer y en una comunidad tlapaneca (Schultze-Jena, 1938).

los rituales actuales. En su libro da argumentos metodológicos en favor del uso de analogías en los estudios iconográficos y construye la base metodológica para la etno-iconología, que después es desarrollada y aplicada por todos los miembros de la escuela holandesa.

Finalmente, en el tercer nivel interpretativo se generan las reflexiones y conclusiones obtenidas de los análisis previos, obviamente en un amplio contexto histórico, social e ideológico de la época pertinente. Es sólo en esta etapa que pueden sugerirse las razones de por qué se hicieron los documentos, por quiénes y cuándo. También es el momento de evaluar el significado de un documento o todo el grupo del que forma parte, dentro de un contexto de procesos políticos e históricos más extenso. 


\section{LA TRADICIÓN MEXICANA}

El gran investigador mexicano de los documentos pictográficos es, obviamente, Alfonso Caso, quien con sus muchos estudios abrió el camino para las interpretaciones modernas de los códices históricos. Caso tenía un claro enfoque en los documentos pictográficos mixtecos y es precisamente allí donde vemos su fuerte influencia a través de Elizabeth Smith y Manuel Hermann Lejarazu, aunque ambos investigadores forman también parte de la escuela holandesa.

No tenemos ningún escrito que explique el método de Caso aplicado en sus estudios, pero hay una clara línea metodológica en las publicaciones de los tres investigadores mencionados que implícitamente concuerda por completo con los tres pasos de la etno-iconología, aunque ésta no fue enteramente aplicada por Caso. Así, en las ejemplares publicaciones de Smith (1973, especialmente pp. 89-161) y Hermann Lejarazu (2003) se comienza con un análisis iconográfico de los glifos (paso 1), seguido de una contextualización de los textos pictográficos (paso 2) para, finalmente, llegar a las conclusiones (paso 3$)^{3}$. El desarrollo de esta manera de trabajar estuvo íntimamente relacionado con el desarrollo del método etnoiconológico de la escuela holandesa, en la cual Smith tenía mucha influencia y de la cual Hermann forma parte.

Curiosamente, no hay más seguidores de Caso en México. Los demás investigadores, que no trabajan con el método Galarza — que trataré más abajo—, son muy diversos en sus enfoques y formas de trabajo ${ }^{4}$. Por lo mismo, cada investigador y cada publicación merece un análisis en sí, pero no hay espacio para ello en este lugar. Sin embargo, en general se puede decir que las publicaciones de los investigadores mexicanos tienen su enfoque en

\footnotetext{
${ }^{3}$ Obviamente, tenemos también los trabajos del gran sabio Luis Reyes García, quien estaba muy relacionado con la escuela holandesa e involucrado en el desarrollo del método de la etno-iconología, razón por la cual le considero parte de esa tradición.

${ }^{4}$ Las más destacadas entre estos investigadores son Carmen Aguilera, Laura Rodríguez Cano, María Teresa Sepúlveda y Herrera, Constanza Vega Sosa y Perla Valle.
}

un documento pictográfico en particular con un estudio descriptivo formal y una contextualización histórica de la respectiva región o periodo relacionado con el documento en cuestión 5 . Tomando en cuenta que los investigadores de esta tradición viven y trabajan relativamente cerca de los pueblos indígenas de donde proceden las pictografías, es extraño que no se haga trabajo de campo para relacionar la información documental con la del paisaje real y la del conocimiento local. En muchos casos, ni siquiera hay estudios de archivo, a pesar de que el Archivo General de la Nación está en la ciudad de México. Esta carencia resulta muchas veces en errores innecesarios o en estudios aceptables, en los que una mayor profundidad y calidad hubiese sido relativamente fácil de conseguir.

Quiero mencionar y destacar el importante proyecto del Colegio Mexiquense que tiene como objetivo publicar ediciones facsimilares de los documentos pictográficos y, particularmente, de los llamados Códices Techialoyan $^{6}$. La calidad de las reproducciones fotográficas es extraordinaria, lo que otorga la oportunidad a los investigadores de trabajar con las fuentes primarias. El legado de este proyecto ha dado como resultado ocho publicaciones de ediciones facsimilares, en las cuales — con algunas excepciones - los comentarios siguen la línea arriba estipulada, tratándose básicamente de descripciones formales?.

Puede decirse que se ha desarrollado una tradición particular, bastante relacionada con el Colegio Mexiquense. Ésta inició con Donald Robertson, autor de varios e importantes trabajos sobre los Códices Techialoyan. Uno de

\footnotetext{
${ }^{5}$ Véase, por ejemplo, Perla Valle (1994a, 1994b), Carmen Aguilera (1981, 1986), Ana Rita Valero (1994), Teresa Sepúlveda (1994, 2005), Constanza Vega (1991) y Xavier Noguez $(1996,1999)$.

${ }^{6}$ En el pasado había proyectos similares de otras instituciones como, por ejemplo, Códices mesoamericanos, del Instituto Nacional de Antropología e Historia (INAH) y la Benémerita Universidad Autónoma de Puebla (BUAP) (Valle, 1994b; Mohar Betancourt, 1994; Sepúlveda y Herrera, 1994; Valero de García Lascuraín, 1994; Yoneda, 1994), o Códices y manuscritos Tlaxcala (Aguilera, 1981, 1986; Códice de Huamantla, 1984).

${ }^{7}$ Noguez y Hernández R., 1992; Harvey, 1993; Valle, 1994a; García Castro, 1999; Noguez, 1999; Ruiz Medrano y Noguez, 2004; Castañeda de
} la Paz, 2006, Martínez García, 2007. 
sus adeptos fue Xavier Noguez, quien desde hace varios años ha estimulado el proyecto de publicar ediciones facsimilares en el Colegio Mexiquense ${ }^{8}$. Recientemente el relevo de esta tradición lo ha tomado Raymundo Martínez García, adepto de Xavier Noguez.

\section{LA TRADICIÓN AMERICANA}

La tradición americana del estudio de los documentos pictográficos tiene su origen en dos investigadores principales: George Kubler y Douglas Frazier, si bien H. B. Nicholson - aunque de la segunda generación — inspiró asimismo a muchos investigadores dentro de esta disciplina. Los tres fueron historiadores del arte y desde la década de 1950 publicaron una gran serie de artículos y libros que ha servido para formar o inspirar a casi todos los investigadores de los documentos pictográficos que están activos hoy día en Estados Unidos. Como una segunda generación de investigadores podríamos mencionar a Elizabeth Smith (de la que ya se habló en relación con la escuela holandesa), Donald Robertson, H. B. Nicholson y Cecilia Klein, seguidos por una gran tercera generación de investigadores como Barbara Mundy, Elizabeth Hill Boone, Eloise Quiñones Keber y Ellen Baird. Recientemente se está formando una nueva generación integrada por jóvenes investigadores, como Delia Cosentino y Lori Diehl. En este punto es importante notar que, salvo algunas excepciones, todas son historiadoras del arte, aspecto que determina en gran medida el tipo de investigación que han producido.

Aunque Nicholson (1976) ha dejado una fuerte declaración metodológica relacionada con la interpretación y lectura de los documentos pictográficos, los principales estudios de la tradición americana son, por un lado, descripciones formales, preocupaciones de estilo o de ciertos elementos pictográficos y el desarrollo de éstos a través de la Colonia (principalmente la tercera generación); y, por otro lado, obras globales de cierto tipo de documen-

${ }^{8}$ Para otras importantes publicaciones de este autor véanse Noguez, 1995 y 1996. to o de documentos de cierta región (principalmente la cuarta generación). Es importante notar que no hay especialistas - tal vez con la excepción de Elizabeth Hill Boone- que dediquen exclusivamente sus esfuerzos a las investigaciones de los documentos pictográficos. Más bien, en Estados Unidos se estudia la iconografía mesoamericana o la cultura visual mexicana —que también incluye las pictografías indígenas - para llegar a grandes generalizaciones 9 .

Sin menospreciar las importantes contribuciones de la tradición americana, es sorprendente notar la poca atención dedicada al método y al trabajo de campo. Dado que la mayoría de las publicaciones están dirigidas hacia las generalizaciones o patrones globales, los investigadores entran muy pocas veces en la discusión e interpretación de documentos individuales, algo que dificulta la evaluación de las bases sobre las cuales construyen sus ideas y conclusiones. Por otro lado, la falta de trabajo de campo es total. Tal vez tiene su origen en la ascendencia intelectual de George Kubler, quien estaba fuertemente en contra de la relación entre los pueblos indígenas y los documentos coloniales y prehispánicos. Sin embargo, hay suficiente evidencia de que, tanto en las investigaciones de los documentos mánticos/religiosos, como en los históricos-geográficos, el trabajo de campo enriquece y mejora los estudios y los resultados.

\section{LA ESCUELA ESPAÑOLA}

La escuela española es muy reciente y no ha cristalizado todavía. El primer interés en España por los documentos pictográficos comenzó con José Alcina Franch, quien elaboró varios índices, pero nunca abordó la interpretación.

\footnotetext{
${ }^{9}$ Parece que los estudios de los documentos pictográficos están en parte determinados por la organización académica institucional en Estados Unidos. Los códices mesoamericanos son considerados productos visuales del arte y, por tanto, no son estudiados dentro de los Departamentos de Historia, sino dentro de los de 'Arte' y, actualmente, dentro de los de 'Estudios Latino Americanos'. Esa práctica apunta a -incluso refuerza - una valoración, en mi opinión, equivocada de los documentos alfabéticos como históricos y de los documentos pictográficos como no-históricos.
} 
Este último aspecto fue iniciado seriamente por Juan José Batalla Rosado, quien trabaja principalmente con documentos del centro de México y para los cuales está desarrollando el llamado método científico que, según el mismo autor, consiste de tres pasos:

[... en primer lugar el estudio codicológico y diplomático de los documentos, y en segundo lugar el análisis del contenido, diferenciando en aquellos casos que lo contengan el Libro Indígena (pinturas) del Libro Escrito Europeo (texto explicativo alfabético), para posteriormente llevar a cabo su estudio conjunto. Una vez fijados los resultados es cuando se deben comparar los mismos con todo tipo de fuentes relacionadas con el tema objeto de estudio, contextualizando la información obtenida (Batalla Rosado y Jansen, 2006: 65).

Especialmente llamativo es el énfasis en la codicología ${ }^{10}$ y la distinción entre el libro indígena y el libro escrito europeo. Este último aspecto tiene su origen en el objeto principal de estudio de Batalla Rosado: el Códice Tudela. Se trata de un documento pictográfico de naturaleza mántica con numerosos textos añadidos por varios autores, y que forma parte de una serie de copias y reelaboraciones del llamado Grupo Magliabecchiano.

Los documentos mánticos son documentos usados en Mesoamérica para la adivinación y, como tales, estaban íntimamente relacionados con la religión indígena. Es precisamente en ese aspecto que la sociedad indígena sufrió grandes cambios con la llegada de los españoles y el sistema religioso católico. Por tanto, no debe sorprender que, a menudo, los textos o las glosas añadidas a los dibujos muestren un desconocimiento (parcial) del significado de éstos. Es probable que dichos textos fueran escritos: 1) por europeos que no conocieron la religión mesoamericana; 2) por indígenas que no quisieron mostrar su conocimiento de la religión mesoamericana por razones de posibles represalias, o 3) por indígenas que habían su-

\footnotetext{
${ }^{10}$ La codicología es la disciplina que estudia manuscritos en su aspecto material y consiste, por ejemplo, en una determinación del orden y la cantidad de los cuadernillos, un estudio del papel por medio de las filigranas, una determinación de los escribanos que estaban involucrados en la producción del documento por medio de los textos mismos y de la foliación, entre otros elementos.
}

frido una aculturación respecto a la religión mesoamericana y habían perdido, así, el conocimiento de la misma. Por tanto, para tratar los documentos mánticos que contienen glosas es necesario tener mucho cuidado al usar dichos textos para la interpretación de los dibujos. Es por eso que, con justa razón, Batalla Rosado trata las pinturas (el libro indígena) separadamente de los textos alfabéticos (el libro escrito europeo).

Sin embargo, un método no es una receta que se puede aplicar mecánicamente a cualquier documento. Cada texto es único y cada contexto en el que fue elaborado ha dado lugar a una relación significativa única entre los elementos pictográficos que constituyen el texto. Mientras que en los documentos mánticos la relación entre las pinturas y los textos alfabéticos explicativos puede haber sido rota por la aculturación y la represión religiosa, en la mayoría de los documentos pictográficos mundanos no existe tal ruptura. Consecuentemente, y en general, las glosas y los textos añadidos a las pictografías mundanas son fundamentales para el entendimiento del mensaje pictográfico. Por ello, la separación metodológica de las pinturas y los textos alfabéticos en los documentos pictográficos mundanos puede ser contraproducente y conducir a discursos innecesariamente largos ${ }^{11}$.

Finalmente, es importante notar que el estudio codicológico debe ser un paso en el análisis hacia un mejor entendimiento de un documento. El objetivo siempre tiene que ser un análisis histórico y culturalmente contextualizado. Sin embargo, en muchas publicaciones en las que se aplica la codicología parece que este paso metodológico se ha convertido en la meta ${ }^{12}$.

\section{LA ESCUELA GALARCISTA}

El método Galarza, que cuenta con un gran número de adeptos en Francia, Italia y México, nació de una gran preocupación por la falta de 'reconocimiento de la existencia de la escritura en las culturas indígenas' (Mohar

\footnotetext{
${ }^{11}$ Véase, por ejemplo, Ruz Barrio, 2006.

12 Véase especialmente Batalla Rosado 1999a, 1999b, 2001, 2002, 2003.
} 
Betancourt y Hernández Díaz, 2006: 10) ${ }^{13}$. Es por eso que desde la década de 1960 Joaquín Galarza buscó y desarrolló una manera de leer los documentos pictográficos mesoamericanos - aunque principalmente de los grupos nahuas- como si fueran textos fonético-silábicos. Para llegar a esta meta, Galarza y sus seguidores han elaborado un análisis que consiste de seis pasos:

1. Segmentación sistemática de los grupos gráficos o plásticos siguiendo el orden de composición del texto expuesto por el tlacuilo mismo.

2. Una segunda segmentación, en la que se separan los elementos mínimos del sistema para examinarlos sintagmática y paradigmáticamente en relación con otros elementos del texto; primero por comparación visual.

3. Extracción del valor fonológico revelado por el análisis estilístico, que esclarece el contenido temático del que están cargados los elementos plásticos. Relacionar los elementos mínimos directamente con la lengua, con base en el significado y la lectura, tomando en cuenta la polivalencia y polifunción de cada elemento.

4. Elaboración de una primera lectura fonológica-denotativa y sintáctica de cada grupo para reconstruir oraciones y/o párrafos del texto en náhuatl —y establecer

13 Tomando en cuenta los criterios en la que está basada esta preocupación, cabe la pregunta de si es necesaria. En el siglo XIX se desarrollaba, dentro del modelo de la evolución cultural, la definición de lo que era una civilización con base en la existencia de ciertas características culturales. Una característica importante era precisamente la escritura. Los grupos culturales con un sistema de escritura eran civilizados y los que no la tenían (todavía) no lo eran. Aunque este modelo y su definición de civilización ya no es aceptado en el mundo académico, varias de sus ideas siguen todavía implícitas en los discursos y los pensamientos de algunos investigadores actuales. Es por esta razón que muchos investigadores quieren a toda costa que el grupo cultural que investigan tenga un sistema de escritura. La definición de escritura más aceptada establece una relación entre la forma gráfica y las oraciones, pero los sistemas mesoamericanos no caben en esa definición. Hay entonces dos tendencias para incluir estos sistemas pictográficos en la definición de escritura ( $y$ con ella, en la de civilización). Una es ampliar la definición de escritura (Elizabeth Boone, Javier Urcid, etc.); la otra, forzar los sistemas mesoamericanos dentro de la definición existente (Joaquín Galarza). Sin embargo, si no nos preocupáramos por la definición de civilización y simplemente aceptáramos los sistemas pictográficos mesoamericanos como sistemas gráficos de comunicación, no necesitaríamos extender la definición de escritura ni tendríamos que forzar los sistemas mesoamericanos para que tuvieran cabida dentro de una definición que nunca fue desarrollada para los sistemas de América. su traducción al español— que corresponden a las asociaciones plásticas de los elementos mínimos.

5. Elaboración de una segunda lectura connotativa y metafórica de cada grupo, de los conjuntos, tomando en cuenta su polivalencia y polifuncionalidad.

6. Elaboración de una tercera lectura global del relato (síntesis de las lecturas parciales, siguiendo las reglas del idioma náhuatl).

Con base en estos pasos queda claro que el sustento del análisis es la suposición de que los elementos mínimos de las pictografías tienen un valor fonético y que éstos están organizados de tal manera que forman oraciones. Obviamente esta suposición está basada en la definición clásica de la escritura que, precisamente, relaciona la forma gráfica con la lengua y la formulación de oraciones. Sin embargo, aparte de los sistemas maya, epi-olmeca y zapoteco clásico, no tenemos ninguna evidencia de que los otros sistemas pictográficos mesoamericanos tengan esa relación directa con la lengua. Entre los investigadores hay un consenso de que los documentos pictográficos — por lo menos los de carácter histórico-geográficoestán fuertemente relacionados con la oralidad, pero de ninguna manera representan un sistema silábico-fonéti$\mathrm{Co}^{14}$. Entonces, si los sistemas pictográficos mesoamericanos no son representaciones directas de una lengua, la base teórica y metodológica del método Galarza no existe.

Además, sabemos que el significado de los distintos signos procede de las relaciones mutuas y significativas entre los mismos y que, por tanto, una lectura no se deriva del nombramiento de todos los elementos de manera individual, sino del análisis de las relaciones entre estos elementos. Esto quiere decir que cada lectura siempre es contextual. El contexto, entonces, limita las posibles lecturas, ya que cada elemento ocurre dentro de una unidad temática que determina cómo leer los elementos.

Relacionando lo anterior con los dos primeros pasos del método Galarza, que tienen como objetivo separar los elementos mínimos del sistema, observamos que pre-

\footnotetext{
${ }^{14}$ Siendo la principal excepción los glifos toponímicos y onomásticos.
} 
cisamente la abstracción de los elementos pictográficos de su contexto produce los errores, o más bien, la imposibilidad de llegar a una lectura. Un problema de este método es el rompimiento que ocurre entre los catálogos como verdadera meta del método y la articulación de esos catálogos con el mensaje del documento. El catálogo es un ejercicio bueno, pero después es necesario avanzar en las relaciones entre los signos antes de llegar a una lectura. No es posible conectar una lectura de signos sueltos, ya que eso resulta en una ilimitada cantidad de posibilidades, incluso posibilidades ridículas.

Un ejemplo revelador lo tenemos en el artículo de Mohar Betancourt y Hernández Díaz (2006) en el que explican el método Galarza. Su figura 12 muestra una ficha de trabajo que trata de una "lectura (hipotética) global propuesta en mexicano para el glifo en cuestión”, en alusión al muy conocido glifo de Chapultepec:
Como podemos ver, las autoras llegan a una lectura de Chapoltepec Ameyalco o "Cerro de Chapulines donde abunda o nace el agua". Pero Chapultepec es un lugar muy conocido de las fuentes históricas y en ninguna de éstas tenemos una referencia a la parte de Ameyalco. Galarza y sus seguidores explican ese fenómeno por la "desaparición, a raíz del nuevo orden colonial, del conocimiento generado por aquellos personajes, cuya especialidad era leer en esos lienzos o pliegos de papel amate todo lo que se consideraba necesario registrar" (ibid.: 12-13). Sin embargo, este desprecio hacia las fuentes indígenas alfabéticas y a favor de los documentos pictográficos no es compartido por otros investigadores ${ }^{15} \mathrm{y}$ no puede ser aceptado como explicación para la falta de una referencia a Ameyalco. Parece entonces más probable que la lectura propuesta no es la correcta y que el elemento glífico del agua simplemente no se leía, como es muy común en la pictografía mesoamericana.

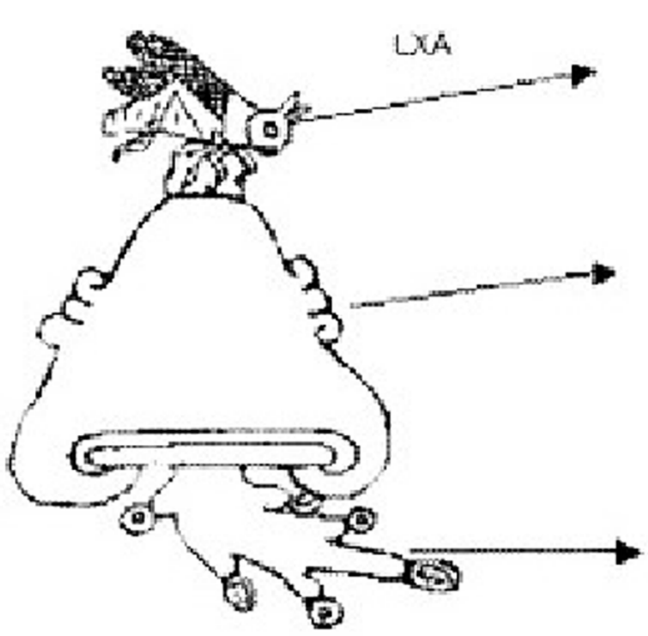

\section{Chapollin = saltamontes o chapulín}

$$
\text { Tepetl }=\text { cerro }
$$

\section{Ameyalli $=$ manantial}

Chapoltepec Ameyalco = "Cerro de chapuliness donde abunda o nace el agua" Lugar, asentamiento, población situada junto a un cerro de donde brota un manantial y en donde abundan los chapulines.

${ }^{15}$ Véase, por ejemplo, Kirchhoff et al., 1989; Reyes García et al., 1996; Reyes García, 2001; Tena, 2002; Johansson, 2004. 
A partir de este ejemplo se podría decir que estamos ante una aberración de los seguidores de Galarza, pero otro ejemplo de la misma índole, realizado por el propio Galarza, revela que no es así. En un libro infantil hecho por Galarza y Libura (2002: 18) tenemos el siguiente ejemplo: huacan?" Aparte de que no hay ninguna glosa al lado del glifo que diga Colhuacan, la propuesta tiene como consecuencia que no se puedan usar la mayoría de las fuentes indígenas porque, según Galarza y sus seguidores —los autores de, por ejemplo, la Historia Tolteca-Chichimeca

Podemos descifrar el nombre del lugar donde se encuentra la cueva.

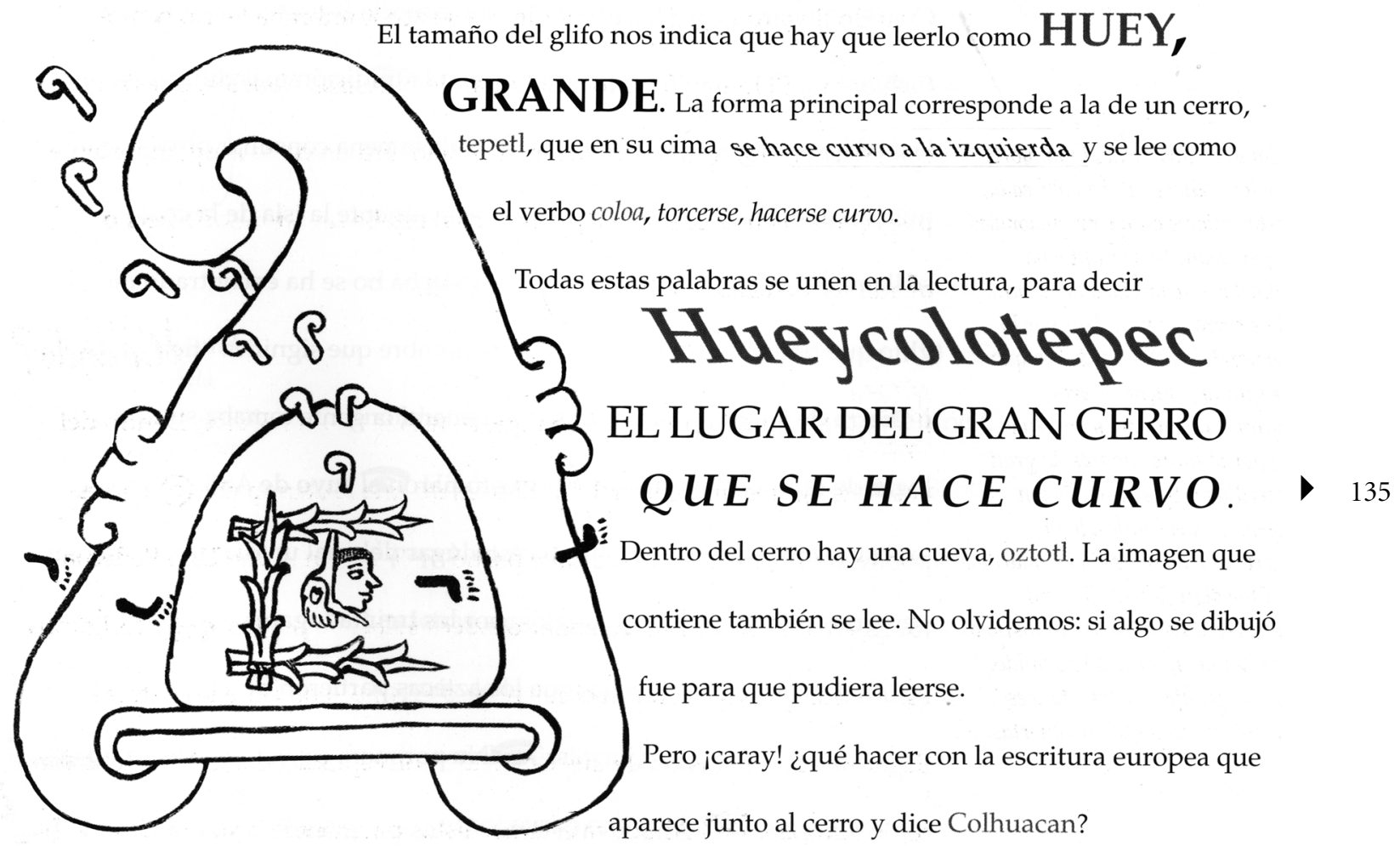

La lectura que los autores generan es la de Hueycolotepec o "Lugar del gran cerro que se hace curvo". Pero, de nuevo, no tenemos ninguna referencia a ese lugar en las múltiples fuentes históricas que han sobrevivido hasta nuestros días. Sin embargo, el topónimo representado es muy conocido, ya que se trata de Culhuacan o Teoculhuacan, un lugar muy importante en la peregrinación mexica y en la cosmovisión mesoamericana. En seguida los autores se preguntan: "Pero ¡caray!, ¿qué hacer con la escritura europea que aparece junto al cerro y dice Col- o de la Historia de los mexicanos por sus pinturas—, eran autores europeos. La propuesta es absurda, por lo que es más apropiado ignorar la lectura del glifo con base en lo dicho arriba.

\section{CONCLUSIÓN}

El estudio de los documentos pictográficos se puede dividir en cinco tradiciones y escuelas diferentes, de las cua- 
les he discutido sus respectivos métodos y sus problemas. Con excepción de la escuela galarcista, todas las tradiciones son, de una u otra manera, compatibles y complementarias. Donde la escuela holandesa tiene su enfoque en la contextualización histórica y cultural de (grupos de) documentos específicos, la tradición americana busca más bien las generalizaciones sobre los documentos como representaciones de un género o de una región. La escuela española sigue en gran parte a la holandesa, pero su enfoque se centra en el análisis del material de los documentos y en la manera en que fue producido el texto glífico y alfabético. Finalmente, la tradición mexicana tiene como objetivo principal la publicación de los documentos como fuentes para el estudio, estableciendo así la base para todos los demás análisis.

Obviamente, hay muchos más investigadores que los arriba mencionados, pero la mayoría no caben muy bien dentro de una u otra tradición o escuela. Se pueden mencionar a los historiadores Patrick Lesbre o Jerome Offner, quienes en sus trabajos usan —y con mucha razón— los documentos pictográficos como un texto histórico más, historia texcocana. Por otro lado, hay también muchos investigadores cuyas investigaciones son ajenas al estudio estrictamente pictográfico, pero que utilizan elementos iconográficos procedentes de los códices. Un claro ejemplo es Alfredo López Austin y sus adeptos, que trabajan principalmente con la cosmovisión indígena.

No obstante, cada vez es más evidente que para el uso intensivo y múltiple de las pictografías es esencial un profundo estudio de los documentos pictográficos. Por tanto, tenemos que reconocer que dicho estudio es una disciplina en sí misma. Aunque íntimamente relacionada con otras disciplinas como la historia, la lingüística o la filología, por sólo mencionar algunas, se pueden llegar a cometer graves errores si no se está formado en la lectura e interpretación de los códices. Los documentos pictográficos no son simples comics que cualquier investigador puede leer. Un buen análisis pictográfico requiere una sólida y especializada formación, un método específico y ciertos conocimientos de otras disciplinas relacionadas con la que aquí nos atañe. El niño se ha convertido en un adolescente y está pidiendo que lo tomen en serio.

\section{Bibliografía}

Acuña, René (ed.), 1984, Relaciones geográficas del siglo XVI: Tlaxcala, t. II, Universidad Nacional Autónoma de México, México.

Aguilera, Carmen, 1981, El Tonalamatl Aubin, Gobierno del Estado de Tlaxcala, Tlaxcala (Tlaxcala, Códices y manuscritos ,1).

— 1986, Lienzos y códice de Tepeticpac. Estudio iconográfico e histórico, Gobierno del Estado de Tlaxcala, Tlaxcala (Tlaxcala, Códices y manuscritos, 4).

Asselbergs, Florine G. L., 2004, Conquered Conquistadors. The Lienzo de Quauhquechollan: A Nahua Vision of the Conquest of Guatemala, CNWS Publications, Research School CNWS, Leiden.

Batalla Rosado, Juan José, 1999a, "Estudio codicológico del Códice Tudela", en Anales del Museo de América, núm. 7, pp. 7-63.

_ 1999b, "Resultados del estudio de la paginación del Códice Tudela, documento colonial azteca realizado en el siglo XVI", en Actas del III Congreso Nacional de Historia del Papel en España, Consellería de Cultura, Educació i Ciéncia de la Generalitat Valenciana, Valencia, pp. 83-112.

__ 2001, "Tintas utilizadas por el amanuense del Libro Escrito Europeo del Códice Tudela", en Anales del Museo de América, núm. 9, pp. 219-254.

—_, 2002, Códice Tudela y el Grupo Magliabechiano: La tradición medieval europea de copia de códices en América, Ministerio de Educación Cultura y Deportes, Madrid (Colección Thesaurus Americae, 4).

_ 2003 , "Estudio formal y codicológico del Códice $\mathrm{Ca}$ bezón o Códice de Costumbres y Fiestas, enterramientos y diversas formas de proceder de los indios de Nueva Espa$\tilde{n} a$ ", en Actas del V Congreso Nacional de Historia del Papel en España, CCG Ediciones, Girona, pp. 105-114.

— y Maarten Jansen, 2006, "Introducción", en Revista Española de Antropología Americana, vol. 36, núm. 2, pp. 65-67.

Castañeda de la Paz, María, 2006, Pintura de la Peregrinación de los Culhuaque-Mexitin (El Mapa de Sigüenza). Análisis de un documento de origen tenochca, El Colegio Mexiquense, Instituto Nacional de Antropología e Historia, Zinacantepec.

Códice de Huamantla, 1984, Códice de Huamantla. Manuscrito de los siglos XVI y XVII, Gobierno del Estado de Tlaxcala, Tlaxcala.

Doesburg, Sebastián van, 2001, Códices cuicatecos; Porfirio Díaz y Fernández Leal, Gobierno Constitucional del Estado de Oaxaca, Miguel Ángel Porrúa, México. 
Galarza, Joaquín y Krystyna M. Libura, 2002 [1999], Para leer La Tira de la Peregrinación, Ediciones Tecolote, México.

García Castro, René, 1999, Códice Xiquipilco-Temoaya y títulos de tierras otomíes. Asentamientos, documentos y derechos indigenas en conflicto, siglos XVI-XVIII, El Colegio Mexiquense, Estado de México, Zinacantepec.

Harvey, Herbert R., 1993, Códice Techialoyan de Huixquilucan (Estado de México), El Colegio Mexiquense, Gobierno del Estado de México, Toluca.

Hermann Lejarazu, Manuel, 2003, Códice Muro. Un documento mixteco colonial, Gobierno Constitucional del Estado de Oaxaca, Conaculta, Instituto Nacional de Antropología e Historia, México.

Huamantla, Códice de, 1984, Códice de Huamantla, Gobierno del Estado de Tlaxcala, Tlaxcala, (Tlaxcala, Códices y manuscritos, 4).

Jansen, Maarten, 1982, Huisi Tacu; estudio interpretativo de un libro antiguo: Codex Vindobonensis Mexicanus I, CEDLA, Amsterdam.

__ 1987, "Dzavuindanda, Ita Andehui y Iukano: historia y leyenda mixteca", en Boletín de Estudios Latinoamericanos y del Caribe, vol. 42, Amsterdam, pp. 71-89.

_ 1988, "The Art of Writing in Ancient Mexico: An Ethno-Iconological Perspective”, en Visible Religion. Annual for Religious Iconography, vol. 6, pp. 86-113.

— 1996 , "Lord 8 Deer and Nacxitl Topiltzin", en Mexicon, vol. 18, núm. 2, pp. 25-29.

Johansson, Patrick, 2004, La palabra, la imagen y el manuscrito: Lecturas indígenas de un texto pictórico en el siglo XVI, Instituto de Investigaciones Históricas, Universidad Nacional Autónoma de México, México (Serie Cultura Náhuatl Monografías, 29).

Kirchhoff, Paul, et al., 1989 [1976], Historia tolteca-chichimeca, Centro de Investigaciones y Estudios Superiores en Antropología Social, México.

Kubler, George, 1961, "On the Colonial Extinction of the Motifs of Pre-Columbian Art”, en S.K. Lothrop (ed.), Essays in Precolumbian Art and Archaeology, University of Cambridge Press, Cambridge, pp. 14-34.

_ 1981, "Period, Style and Meaning in Ancient American Art”, en John A. Graham (ed.), Ancient Mesoamerica: Selected Readings, Peek Publications, Palo Alto, pp. 11-23.

Loo, Peter van der, 1982, "Rituales con manojos contados en el Grupo Borgia y entre los tlapanecos de hoy día”, en Coloquio Internacional Los indígenas de México en la época prehispánica y en la actualidad, Rijksmuseum voor Volkenkunde, Leiden, pp. 232-243.

- 1987, Códices, costumbres, continuidad. Un estudio de la religión mesoamericana, tesis de doctorado, Universidad de Leiden.
Martínez García, Raymundo, 2007, Códice Techialoyan de San Francisco Xonacatlán (Estado de México), Colegio Mexiquense, Gobierno del Estado de México, Zinacantepec.

Mohar Betancourt, Luz María, 1994, Mapa de Coatlichan. Códices Mesoamericanos II, Instituto Nacional de Antropología e Historia, Benemérita Universidad Autónoma de Puebla, México.

—_ y Rita Fernández Díaz, 2006, "El estudio de los códices”, en Desacatos, núm. 22, Centro de Investigaciones y Estudios Superiores en Antropología Social, México, pp. 9-36.

Nicholson, H. B., 1976, "Preclassic Mesoamerican Iconography from the Perspective of the Postclassic: Problems in Interpretational Analysis", en H. B. Nicholson (ed.), Origins of Religious Art and Iconography in Preclassic Mesoamerica, University of California, Los Ángeles, pp. 157-173.

Noguez, Xavier, 1995, Códice de Huexotzinco, Coca-Cola, Ediciones Multiarte, Library of Congress, México.

_ 1996, Tira de Tepechpan. Códice colonial procedente del Valle de México, 2 vols., Biblioteca Nezahuacóyotl, México.

__, 1999, Códice Techialoyan de San Pedro Tototepec (Estado de México), El Colegio Mexiquense, Estado de México, Zinacantepec.

—— y Rosaura Hernández R., 1992, Códice Techialoyan García Granados, El Colegio Mexiquense, Gobierno del Estado de México, Toluca.

Nowotny, Karl Anton, 1961, Tlacuilolli: Die mexikanischen Bilderhandschriften. Monumenta Americana III, Verlag Gebr. Mann, Berlin. [Véase también la traducción al inglés: Tlacuilolli: Style and Contents of the Mexican Pictorial Manuscripts with a Catalog of the Borgia Group, University of Oklahoma Press, Norman, 2005.]

Oettinger, Jr., Marion y Fernando Horcasitas, 1982, “The Lienzo of Petlacala. A Pictorial Document From Guerrero, Mexico", Transactions of the American Philosophical Society, vol. 72, núm. 7, American Philosophical Society, Philadelphia.

Oudijk, Michel R., 2000, Historiography of the Bènizàa: The Late Postclassic and Early Colonial Periods, Research School CNWS, Leiden (CNWS Publications, 84).

Reyes García, Luis, 2001, ¿Cómo te confundas? ¿Acaso no somos conquistados? Anales de Juan Bautista, Centro de Investigaciones y Estudios Superiores en Antropología Social, Biblioteca Lorenzo Boturini, México.

— et al., 1996, Documentos nahuas de la ciudad de México del siglo XVI, Centro de Investigaciones y Estudios Superiores en Antropología Social, Archivo General de la Nación, México. 
Roskamp, Hans, 1998, La historiografía indígena de Michoacan. El Lienzo de Jucutácato y los Títulos de Carapan, Research School CNWS, Leiden University, Leiden (CNWS Publications, 72).

Ruiz Medrano, Ethelia y Xavier Noguez, 2004, Códice de Santiago Tlacotepec (Municipio de Toluca, Estado de México), El Colegio Mexiquense, Instituto Mexiquense de Cultura, Zinacantepec.

Ruz Barrio, Miguel Ángel, 2006, "Pintura del pleito entre Tepexpan y Temaxcalapa: estudio preliminar”, en Revista Española de Antropología Americana, vol. 36, núm. 2, pp. 89-109.

Schultze-Jena, Leonhard, 1938, Bei den Azteken, Mixteken un Tlapaneken der Sierra Madre del Sur, Indiana Band III, Jena.

Sepúlveda y Herrera, María Teresa, 1994, Códice de Yanhuitlán. Estudio preliminar, Instituto Nacional de Antropología e Historia, Benemérita Universidad Autónoma de Puebla, México (Códices Mesoamericanos, III).

, 2005, Los lienzos de San Juan Cuauhtla, Puebla, Consejo Nacional para la Cultura y las Artes, Instituto Nacional de Antropología e Historia, México.
Smith, Mary Elizabeth, 1973, Picture Writing from Ancient Southern Mexico. Mixtec Place Signs and Maps, University of Oklahoma, Norman.

Tena, Rafael, 2002, Mitos e historias de los antiguos nahuas, Consejo Nacional para la Cultura y las Artes, México (Cien de México).

Valero de García Lascuráin, Ana Rita, 1994, Códice Cozcatzin, Instituto Nacional de Antropología e Historia, Benemérita Universidad Autónoma de Puebla, México (Códices Mesoamericanos, IV).

Valle, Perla, 1994a, Códice de Tepetlaoztoc (Códice Kingsborough), Estado de México, Colegio Mexiquense, Toluca.

_, 1994b, Códice de Tlatelolco, Instituto Nacional de Antropología e Historia, Benemérita Universidad Autónoma de Puebla, México (Códices Mesoamericanos, I).

Vega Sosa, Constanza, 1991, Códice Azoyú 1: El reino de Tlachinollan, Fondo de Cultura Económica. México.

Yoneda, Keiko, 1994, Mapa de Cuauhtinchan No. 4, Instituto Nacional de Antropología e Historia, Benemérita Universidad Autónoma de Puebla, México (Códices Mesoamericanos, $\mathrm{V})$. 